\title{
MECHANISMS FOR COORDINATION OF MASTER PLANNING AND LOT SIZING WITHIN A HIERARCHICAL PRODUCTION PLANNING MODEL
}

\author{
Rainer Leisten ${ }^{1)}$, Pascal Reusch ${ }^{2)}$ \\ ${ }^{1)}$ University Duisburg-Essen, Leisten@uni-duisburg.de \\ ${ }^{2)}$ University Duisburg-Essen / Arideon GmbH, ReuschP@web.de
}

\begin{abstract}
This paper presents a simulation based analysis of different coordination mechanisms in Hierarchical Production Planning. According to the results of the simulations the effects of Aggregation and Capacity Assignments on the overall costs are discussed.
\end{abstract}

Keywords: Production Planning, Aggregation, Capacity Assignment, Optimization

\section{HIERARCHICAL PRODUCTION PLANNING}

Most modern Production Planning Concepts inherit a hierarchical planning approach. The splitting of a simultaneous production planning approach leads to the reduction of complexity and allows the consideration of organizational structures within the system. ${ }^{1}$

The most popular and widely used paradigm for Hierarchical Production Planning is Manufacturing Resource Planning (MRP II). ${ }^{2}$ It consists of 4 different levels. The highest level is called Master Production Scheduling or Program Planning. It determines a Master Schedule using aggregate capacity limits and demand forecasts. Mathematical Models for Master Planning try to coordinate the use of inventory and overtime.

The next level in the hierarchy has to deal mainly with Lot Sizing Problems. These models have to combine orders for the same product into lots that are produced without interruption. The objective is to minimize the sum of setup and holding costs that have to be paid for inventory that is used to serve orders that are due during the production cycle of a different product or in a period with insufficient production capacity.

The following two levels are Capacity Planning and Production Scheduling which are not going to be analysed here.

\footnotetext{
${ }^{1}$ Okuda (2001): Hierarchical structure in manufacturing systems: a literature survey

${ }^{2}$ Miller (2002): Hierarchical Operations and Supply Chain Planning
}

The use of mathematical models for Production Planning is limited by the complexity of the models that have to be solved. Problems are especially caused by binary and mixed integer restrictions like in a setup constraint of a Lot Sizing Problem. Up to now it is not possible to find optimal solutions for real-world problem sizes. Because of this, companies often use simple heuristics to get feasible solutions for their planning problems. The resulting solutions are usually of poor quality, as the heuristics neglect important constraints of the problem.

Because of these problems a hierarchical planning approach is used to approximate the optimal solution of a model that combines Master Planning and Lot Sizing.

These steps in Production Planning are frequently used, but up till now almost no scientific simulations have shown how to use information or results from one level in the other in order to find a better global solution of the planning process.

The main focus here is to show which mechanisms can be used to coordinate the results of the different levels and how they have to be adjusted such that the result of the hierarchical planning process comes closest to the result of the optimal solution of the total model.

For this purpose a system of models, including mechanisms for coordination, is developed and the performance of the system as well as the quality of the results provided by the system are evaluated by simulations. 


\section{THE SIMULTANEOUS MODEL}

The starting point for the analysis is a simultaneous model that includes the aspects of a Capacitated Lot Sizing Model (CLSP) and a Master Planning Model (MP). I. e., it solves both problems simultaneously, but is computational very demanding, especially for larger problem sizes, due to the binary setup variables.

The mathematical formulation of the problem uses the following symbols:

\section{Parameters:}

- $b_{t}$ regular capacity in period $\mathrm{t}$

- $d_{k t}$ demand for product $\mathrm{k}$ in period $\mathrm{t}$

- $h_{k}$ holding costs for one unit of product $\mathrm{k}$ per period

- $K$ set of products

- $q_{k t}$ lot size for product $\mathrm{k}$ in period $\mathrm{t}$

- $s_{k}$ setup costs per setup for product $\mathrm{k}$

- $T$ lenght of the planning horizon

- $t b_{k}$ capacity consumption for the production of one unit of product $\mathrm{k}$

- $t r_{k}$ capacity consumption for one setup of product $\mathrm{k}$

- $u_{t}$ costs for one unit of additional capacity in period $\mathrm{t}$

Variables:

- $\quad U_{t}$ used additional capacity

- $\quad y_{k t}$ inventory level for product $\mathrm{k}$ in period $\mathrm{t}$

- $\quad \gamma_{k t}$ binary setup variable for product $\mathrm{k}$ in period $\mathrm{t}$

The objective function (1) minimizes the sum of setup costs and inventory holding costs as well as the costs for additional capacity:

$$
\text { Min } Z=\sum_{k=1}^{K} \sum_{t=1}^{T}\left(s_{k} \cdot \gamma_{k t}+h_{k} \cdot y_{k t}\right)+\sum_{t=1}^{T}\left(u_{t} \cdot U_{t}\right)
$$

The inventory balance constraint (2) assures that the demand is met by production or by inventory:

$$
y_{k, t-1}+q_{k t}-y_{k t}=d_{k t} \quad k=1,2, \ldots, K ; t=1,2, \ldots, T
$$

The consideration of the limited capacity is represented by the capacity constraint (3):

$$
\sum_{k=1}^{K}\left(t b_{k} \cdot q_{k t}+t r_{k} \cdot \gamma_{k t}\right)-U_{t} \leq b_{t} \quad t=1,2, \ldots, T
$$

The setup constraint (4) guarantees that if product $\mathrm{k}$ is produced in period $\mathrm{t}$ the binary setup variable has to be set to one in order to charge the right price within the objective function and to keep track of the capacity usage within the capacity constraint:

$$
q_{k t}-M \cdot \gamma_{k t} \leq 0 \quad k=1,2, \ldots, K ; t=1,2, \ldots, T
$$

The remaining constraints state that the inventory start- and end-levels have to be predefined (5), that the decision variables have to be non negative (6) and that the setup variable has to be binary (7):

$$
\begin{array}{lr}
y_{k 0}, y_{k T}=\text { given } & k=1,2, \ldots, K \\
q_{k t}, y_{k t}, U_{t} \geq 0 & k=1,2, \ldots, K ; t=1,2, \ldots, T \\
\gamma_{k t} \in\{0,1\} & k=1,2, \ldots, K ; t=1,2, \ldots, T
\end{array}
$$

\section{MODEL HIERARCHY}

The hierarchy that is deduced from the model above consists of two levels, the Top-Level and the Base-Level. These levels are tied together by several coordination mechanisms. A general framework for this hierarchical connection is shown in Fig. 1.

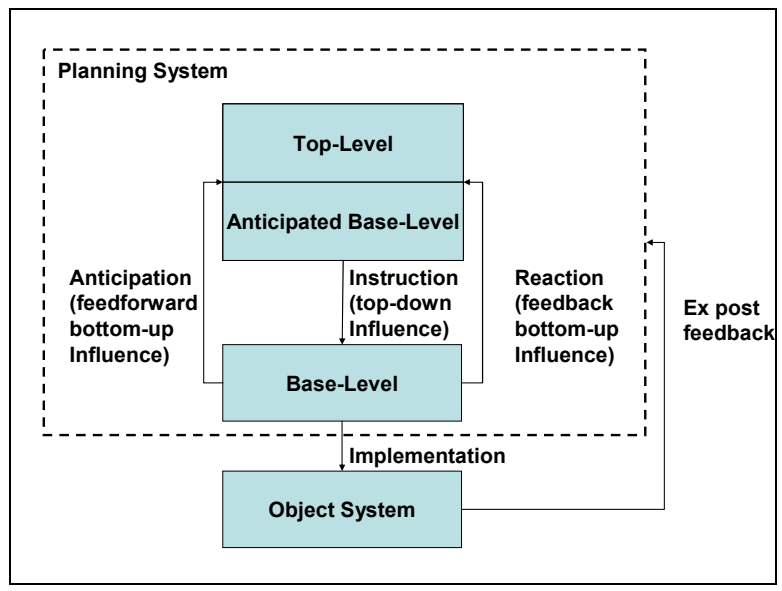

Fig. 1: Hierarchical Planning ${ }^{3}$

Within the planning system the decision problem of the Base-Level is coarsely anticipated by the TopLevel in order to find a better solution for the whole system. After the solution of the Top-Level information is send down to the Base-Level so the Base-Level model can be solved within the frame of the Top-Level solution. Within an iterative approach information can be sent back from the Base-Level to the Top-Level in order manipulate the next solution of the Top-Model. The final solution of the BaseLevel is sent to the Object System to be implemented.

The Top-Level

Within Hierarchical Production Planning the Top-Level usually represents an aggregated view of the problem. Standard ERP Software like mySAP ERP does not include any mathematical programming approach on this level. They propose a "lot for lot" production where every quantity of

${ }^{3}$ Schneeweiss (2003): Distributed Decision Making 
demand is produced in the period where it is requested. Since this strategy is not appropriate for industries with high setup costs and/or high setup times modified approaches are needed.

Advanced Planning Systems like SAP APO offer the possibilities of solving linear or even mixed integer Master Planning models but they do not support the planner to generate the aggregated data that is necessary for this level. Since no aggregation procedures are implemented the user has to define the values manually, which is not acceptable for larger problem sizes. Because there exist no reasonable aggregation procedure the anticipation of the Base-Level is ignored as well.

We use a linear MP model in the Top-Level that does not include any setup decisions. The aggregation procedure is responsible to create the aggregate data in a way that the setup decisions of the Base-Level can be anticipated.

\section{The Base-Level}

Within the Base-Level the remaining setup decision has to be taken. For this problem mixed integer planning models have to be solved, like the Capacitated Lot Sizing Problem. The problem with the usual formulation of the CLSP is that it cannot be solved if the predefined capacity is insufficient. That is why we allow the Base-Level model for additional overtime. The formulation is than equivalent to the formulation of the simultaneous model mentioned above. The only difference is that the model is not solved for all products at once but independently for each product group and the value of $b_{t}$ in (3) is determined by the Top-Level.

\section{METHODS OF COORDINATION}

Within this two-level hierarchy there are two different kinds of coordination. "Bottom-up" coordination represents all information that is transferred from the Base-Level to the Top-Level and "top-down" coordination is based upon all information that is going from the Top-Level down to the Base-Level.

One of the most important factors of "bottom-up" coordination is aggregation. There are two ways of aggregation that can be distinguished - model aggregation and data aggregation. Here the effects of data aggregation are discussed, where independent models solve problems using different levels of data aggregation. The use of model aggregation is going to be analyzed during the sequel of this paper.

\section{Product Aggregation}

A widely used approach to aggregation is the generation of product groups. The main problem within this type of aggregation is the calculation of the aggregated parameters. One way to deduce aggregated demand for a product group is the summation of the demand of the products that belong to the group:

$$
d_{g t}=\sum_{i \in P G_{g}} d_{i t}
$$

Authors like Hax and $\mathrm{Meal}^{4}$ use the mean values of the holding costs and of the capacity consumption of the products belonging to a group to derive the aggregated parameters:

$$
\begin{aligned}
& t b_{g}=\sum_{i \in P G_{g}} \frac{1}{\left|P G_{g}\right|} \cdot t b_{i} \\
& h_{g}=\sum_{i \in P G_{g}} \frac{1}{\left|P G_{g}\right|} \cdot h_{i}
\end{aligned}
$$

The problem of these mean values is that the anticipation of the Base-Level is rather poor. In order to anticipate the setup information from a heuristical presolve of the Base-Level model can be used for the generation of the aggregated data. Here the production quantity $q$ and the inventory level $y$ as well as the average lot size $q^{\text {avg }}$ are used for aggregation:

$$
\begin{array}{r}
t b_{g t}=\sum_{i \in P G_{g}} \frac{q_{i t}}{\sum_{j \in P G_{g}} q_{j t}} \cdot\left(t b_{i}+\frac{t r_{i}}{q_{i}^{a v g}}\right) \\
h_{g t}=\sum_{i \in P G_{g}} \frac{y_{k t}}{\sum_{j \in P G_{g}} y_{j t}} \cdot h_{i}
\end{array}
$$

Besides these Addition Approaches a Work Content Approach, where the demand units are expressed in work content units can be used.

The demand is multiplied by the capacity consumption factor that is adjusted to anticipate the setup capacity requirements before it is summed up to create the aggregate demand:

$$
d_{g t}=\sum_{i \in P G_{g}}\left(t b_{k}+\frac{t r_{k}}{q_{k}^{a v g}}\right) d_{i t}
$$

Because of the transformation of the demand in capacity units the aggregated capacity consumption factor equals unity:

$$
t b_{g}=1
$$

The holding costs of the products are weighed by the ratio of inventory of the product and the amount of inventory for all products of the product group expressed in capacity units before summation:

${ }^{4}$ Hax / Meal (1975): Hierarchical integration of production planning and scheduling 


$$
h_{g}=\sum_{i \in P G_{g}} \frac{\sum_{t=1}^{T} y_{k t}}{\sum_{i \in P G_{g}} \sum_{t=1}^{T}\left(t b_{k}+\frac{t r_{k}}{q_{k}^{\text {qug }}}\right) y_{i t}} \cdot h_{i}
$$

The quality of these three aggregation approaches is influenced by several factors.

1. The size of the product groups. If the product groups contain many products and therefore less groups are needed, the influence of the Top-Level is reduced and the decisions of the Base-Level become more important.

2. The cluster procedure. The cluster procedure determines which products are going to be part of a product group. In our simulations we used two different kinds of cluster procedures. The first procedure aggregates products that have similar capacity requirements. The second one aggregates products that have their first net demand in similar periods. Since there are always products in stock or products are not requested in a period not all products have to be produced during the first period of the planning horizon.

3. Aggregation of initial inventory. There are two ways to generate the aggregate demand parameters. First, the demand and the initial inventory can be aggregated separately. Doing this, the Top-Level has to deal with the initial inventory. Second, the net demand for the products is calculated by subtracting the initial inventory and then the net demand is aggregated.

\section{Top Down Coordination}

Top-Down Coordination here is performed by sending a capacity instruction to the Base-Level. The capacity constraint of the Base-Level model of a product group receives as much capacity as the TopModel determined for the group in each period.

There are two alternatives to Top Down capacity assignment. The Base-Level can either receive only the regular capacity (16) or the regular capacity plus the additional capacity that has been determined by the Top-Model (17):

$$
\begin{aligned}
& I N_{g}=b_{g t}=\left(b_{t}\right) \cdot \frac{t b_{g} \cdot q_{g t}}{\sum_{g^{\prime}=0}^{G} t b_{g^{\prime}} \cdot q_{g^{\prime} t}} \\
& I N_{g}=b_{g t}=\left(b_{t}+U_{t}\right) \cdot \frac{t b_{g} \cdot q_{g t}}{\sum_{g^{\prime}=0}^{G} t b_{g^{\prime}} \cdot q_{g^{\prime} t}}
\end{aligned}
$$

The performance of these different approaches is analysed and evaluated by computer simulations with the intention to show how Hierarchical Production Planning Systems can implement models and model coordination in order to achieve the best possible feasible solution within reasonable time.

\section{CALCULATIONS}

ILOG CPLEX 9.1 $1^{5}$ was used for the calculations and the coordination procedure was implemented in $\mathrm{C}++$. The results represent the average of 10 different automatically generated problem instances.

Random data is generated according to the following rules $(U N(\mathrm{a}, \mathrm{b})=$ Uniform distribution with lower bound a and upper bound $b$ ):

Number of products: $K=300$

Number of periods: $T=12$

Demand: $d_{k t}=49+U N(1,101)$

Capacity consumption factor: $t b_{j k}=U N(1,10)$

Capacity: $b_{t}=1,25 \cdot \frac{\left(\sum_{k=1}^{K} \sum_{t=1}^{T} d_{k t} \cdot t b_{t}\right)}{T}$

Holding costs: $h_{k}=1+U N(1,3)$

Setup costs: $s_{k}=1499+U N(1,1001)$

Costs per unit of additional capacity: $u_{t}=2$

Initial inventory:

$y_{k o}=\left\{\begin{array}{lll}d_{k 1} & f_{b} r & k \leq 0,25 \cdot K \\ d_{k 1}+d_{k 2} & f_{b} r & 0,25 \cdot K<k \leq 0,5 \cdot K \\ d_{k 1}+d_{k 2}+d_{k 3} & f_{b r} & 0,5 \cdot K<k \leq 0,75 \cdot K \\ 0 & f b r & 0,75 \cdot K<k\end{array}\right.$

Setup capacity consumption: $t r_{k}=399+U N(1,201)$

\section{Cluster procedure}

First we tried to determine what kind of cluster procedure and what kind of data aggregation results in the best solution value. The results of the simulation are shown in Fig. 2.

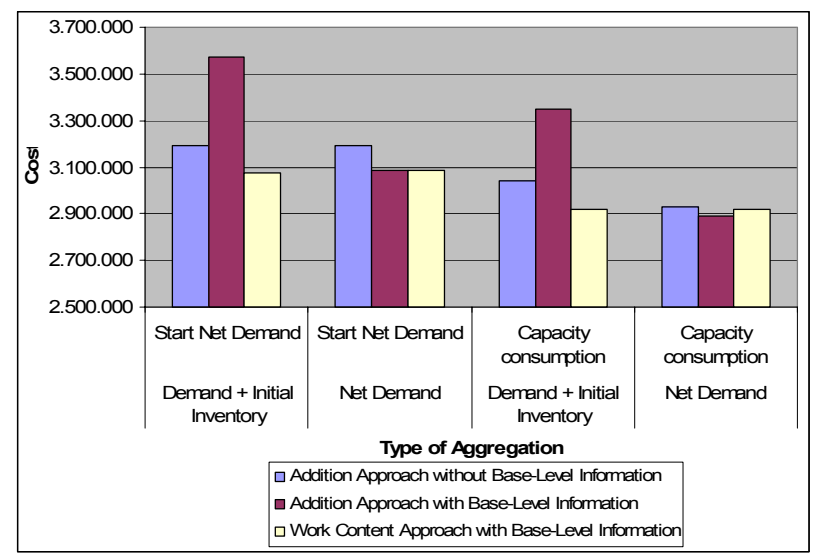

Fig. 2: Effect of different cluster procedures

Independent of the aggregation method a clustering of the products according to the capacity consumption as well as the aggregation of net demand results in the best solution according to total costs. The reason for this is that the separate

${ }^{5}$ http://www.ilog.com/products/cplex/ 
aggregation of demand and inventory allows the Top-Level to use stored items of one product to satisfy demand for a different product in the group. The effect is illustrated in Fig. 3.

The initial inventory of the group is used to cover the demand of the group, even if the group demand results from a different product than the inventory. This results in a Top-Down capacity assignment that does not represent the actual situation and forces the Base-Level to do unnecessary adjustment that result in higher cost.

\begin{tabular}{|c|c|c|c|c|c|}
\hline \multicolumn{6}{|l|}{ Net Demand } \\
\hline & Product 1 & \begin{tabular}{|l} 
Product 2 \\
\end{tabular} & & Group 1 & \multirow{2}{*}{\begin{tabular}{|c|} 
Production \\
Quantity \\
Group 1
\end{tabular}} \\
\hline $\begin{array}{c}\text { Initial } \\
\text { Inventory }\end{array}$ & - & - & $\begin{array}{c}\text { Initial } \\
\text { Inventory }\end{array}$ & - & \\
\hline $\begin{array}{l}\text { Net Demand } \\
\text { Period } 1\end{array}$ & $\mathbf{0}$ & 200 & $\begin{array}{l}\text { Net Demand } \\
\text { Period } 1\end{array}$ & 200 & 200 \\
\hline $\begin{array}{l}\text { Net Demand } \\
\text { Period } 2\end{array}$ & 0 & 200 & $\begin{array}{l}\text { Net Demand } \\
\text { Period } 2\end{array}$ & 200 & 200 \\
\hline $\begin{array}{l}\text { Net Demand } \\
\text { Period } 3 \\
\end{array}$ & 200 & 200 & $\begin{array}{l}\text { Net Demand } \\
\text { Period } 3 \\
\end{array}$ & 400 & 400 \\
\hline \multicolumn{6}{|c|}{ Demand and Initial Inventory } \\
\hline & Product 1 & Product 2 & & Group 1 & \multirow{2}{*}{$\begin{array}{c}\text { Production } \\
\text { Quantity } \\
\text { Group } 1\end{array}$} \\
\hline $\begin{array}{c}\text { Initial } \\
\text { Inventory }\end{array}$ & 400 & 0 & $\begin{array}{c}\text { Initial } \\
\text { Inventory }\end{array}$ & 400 & \\
\hline $\begin{array}{l}\text { Demand } \\
\text { Period } 1\end{array}$ & 200 & 200 & $\begin{array}{l}\text { Demand } \\
\text { Period } 1\end{array}$ & 400 & 0 \\
\hline $\begin{array}{l}\text { Demand } \\
\text { Period } 2 \\
\end{array}$ & 200 & 200 & $\begin{array}{l}\text { Demand } \\
\text { Period 2 }\end{array}$ & 400 & 400 \\
\hline $\begin{array}{l}\text { Demand } \\
\text { Period } 3\end{array}$ & 200 & 200 & $\begin{array}{l}\text { Demand } \\
\text { Period } 3\end{array}$ & 400 & 400 \\
\hline
\end{tabular}

Fig. 3: Effect of separate aggregation of demand and initial inventory

Clustering according to capacity requirements results in better solutions because the Top-Down capacity assignment can be more precise if the products of a group have the same capacity requirements. This way of clustering also groups products with high capacity consumption and thereby the capacity usage of these products can be coordinated by the associated Base-Level model.

Because of these results further calculations all use clustering according to capacity requirements and aggregation of net demand.

Group Size

Fig. 4 shows the effect of group sizes on the solution value.

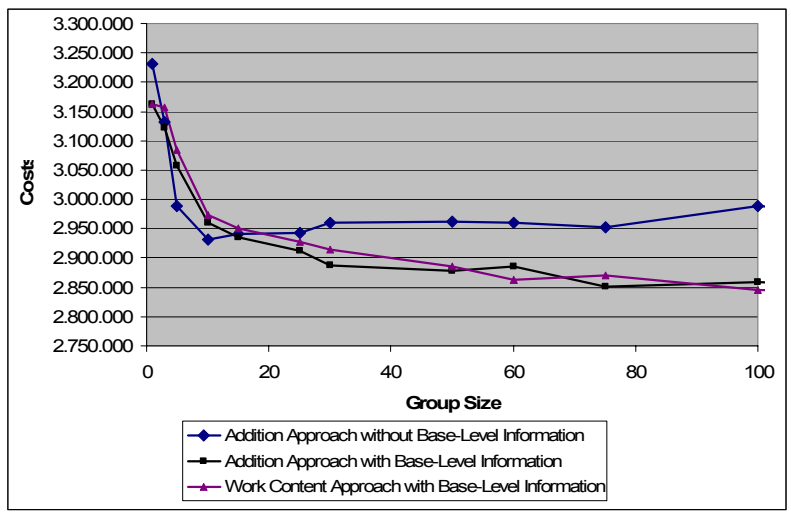

Fig. 4: Effect of different group sizes
The results show that a minimum of 10 products per group should be used. This way the Base-Level can work more accurately which results in a better solution since the model that is solved is more similar to the simultaneous model.

\section{Top Down Coordination}

In regular Production Planning Systems the Master Planning Level is responsible for capacity planning. It usually has a longer planning horizon and therefore it is easier to organize additional capacity.

When we tried to analyse the effect of the assignment of additional capacity by the Master Planning model we found out that the solution is much better when no additional capacity was assigned, as can be seen in Fig. 5. ${ }^{6}$

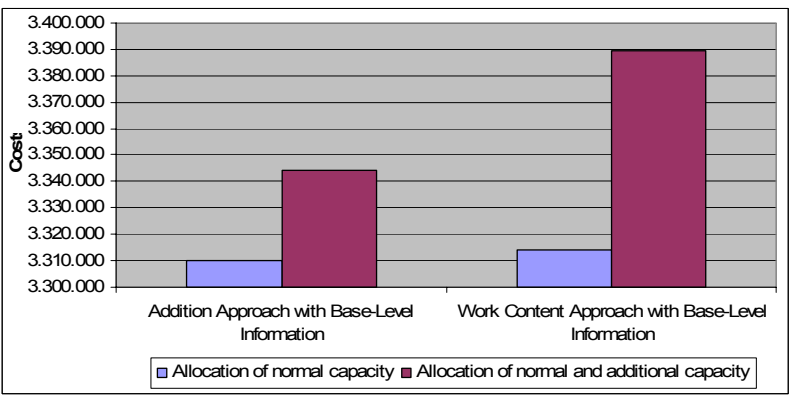

Fig. 5: Effect of different Top-Down capacity assignments on total costs

While the quality of the solution is worse when we assign the additional capacity the time it takes to solve the problem gets reduced, as can be seen in Fig. 6.

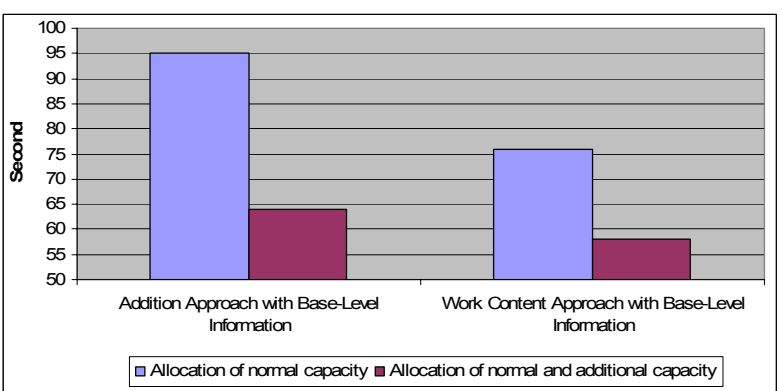

Fig. 6: Effect of different Top-Down capacity assignments on runtimes

One reason for this effect can be that the linear Master Planning Model cannot make setup decisions and therefore assigns the additional capacity to periods where it is not needed once the setup decision is taken into account. The reduced computing time can be explained by the complexity of the Base-Level models. Since the main part of the optimization time is needed to solve the mixed integer models and additional capacity for those

${ }^{6}$ Considering that the costs for additional capacity on the Top-Level is equal to the costs for additional capacity on the Base-Level. 
models reduces their complexity the runtime for the hierarchical system gets reduced significantly.

\section{Effect of machine utilization}

In the beginning of the analysis we only worked with a predefined utilization level of about $80 \%$. Since overtime and additional capacity is not often used if utilization is so low the performance of the aggregation procedures was analyzed with different levels of utilization.

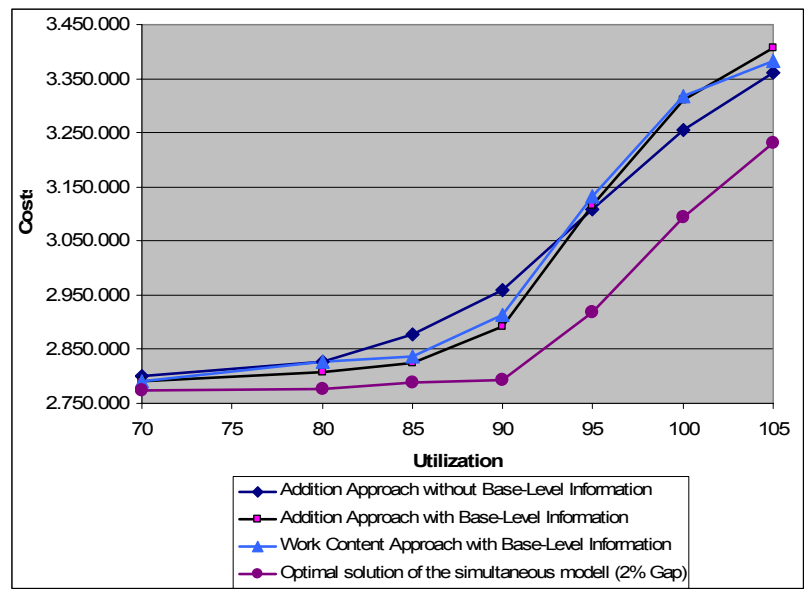

Fig. 7: solution values of different approaches with different machine utilizations

One not very surprising result of this was that the solution value of the simultaneous model is always better than the solution values of a hierarchical approach.

A different effect that was discovered was that the consideration of Base-Level information in the aggregated model resulted in better solution values than the Average Approach only for lower utilization levels. If the utilization is higher than approximately 95\% the Average Approach generates better solutions.

The same performance switch can be observed with respect to runtimes as shown in Fig. 8.

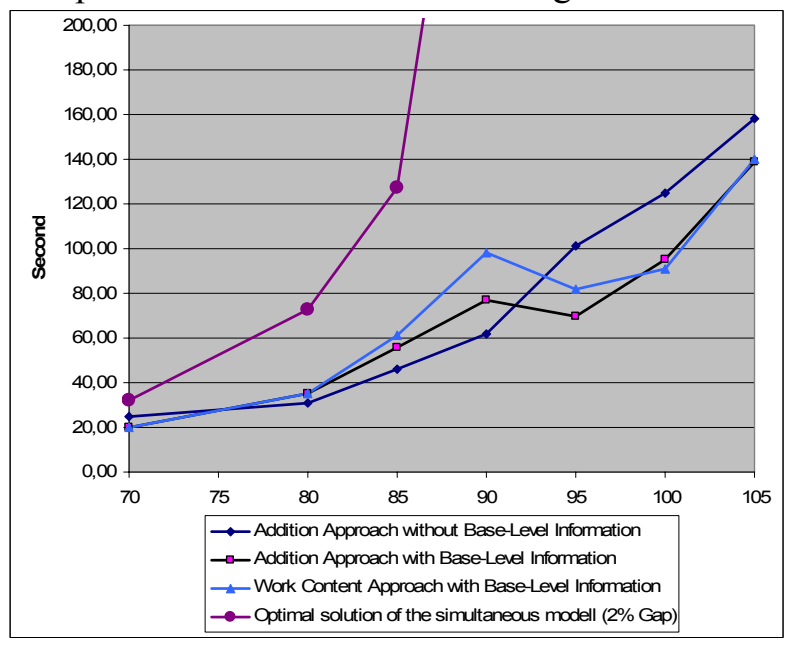

Fig. 8: Runtimes of different approaches with different machine utilizations
The computing time increases with increasing machine utilization. For low utilization levels the Addition Approach without Base-Level Information is faster, but at some point between $90 \%$ and $95 \%$ utilization the performance switches and the approach with Base-Level Information is faster than the one without.

\section{NEXT STEPS}

Several aspects of the hierarchical system still have to be analyzed:

1. What is the effect of using mixed integer models in the Top-Level?

2. Can the ideas of LP-Aggregation be adopted to find better solutions? LP-Aggregation tries to find weights with which the variables and/or constraints of the original problem that are supposed to form a group can be weighed. The resulting reduced problem has the same structure as the original problem, but is of much smaller size. The weights can be calculated in an iterative procedure using primal and dual information of the solution of the aggregated problem to adjust the weights. ${ }^{7}$ The problem is that dual information is not accessible for mixed integer problems and must be approximated somehow.

3. What is the effect of different costs for additional capacity in different levels of the hierarchy?

\section{REFERENCES}

[1]. Hax / Meal (1975): Hierarchical integration of production planning and scheduling; in Geisler: Logistics, TIMS Studies in Management Science, Volume 1, Amsterdam, pp. 53-69.

[2]. Miller (2002): Hierarchical Operations and Supply Chain Planning, Berlin

[3]. Okuda (2001): Hierarchical structure in manufacturing systems: a literature survey; in International Journal of Manufacturing Technology and Management - Volume 3, No.3 pp. 210-224

[4]. Schneeweiss (2003): Distributed Decision Making, Berlin

[5]. Leisten (1998): An LP-aggregation view on aggregation in multi-level production planning; in Annals of Operations Research Volume 82, No. 1, pp. 413-434(22)

\footnotetext{
${ }^{7}$ Leisten (1998): An LP-aggregation view on aggregation in multi-level production planning
} 


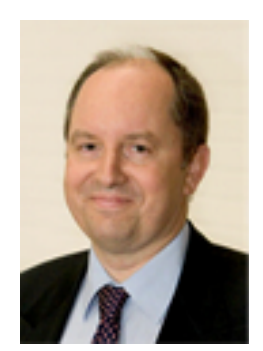

Professor Dr. Rainer Leisten was born in 1957 and gained a Diploma in mathematics with business administration and a Ph.D. in Business Administration from the University of Cologne. His doctoral thesis concerned flow-shop scheduling with limited buffer storage. He has 3 years experience in bank controlling and is currently professor for business administration and production management at the University of Duisburg-Essen, Mercator School of Management, Duisburg, Germany. Dr. Leisten's main research interests are supply chain management, aggregation and complexity in production planning, scheduling, and production and operations management.

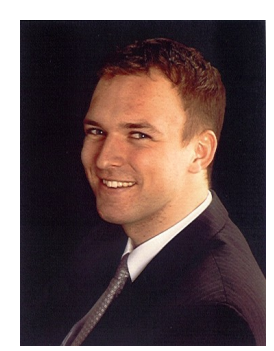

Pascal Reusch gained a diploma in Information Systems from the University of Cologne. He has been working as a research associate at the University of Duisburg-Essen, Mercator School of Management, Duisburg, Germany, writing his doctoral thesis on aggregation and complexity in production planning. Currently he is working as a Management Consultant for Arideon in Duesseldorf, Germany. 